\title{
El control político del Parlamento de Andalucía de las contabilidades de los Grupos parlamentarios y la financiación de los Partidos políticos
}

\author{
Rafael Cano Silva \\ Funcionario del Cuerpo Técnico del Parlamento de Andalucía \\ r.cano@parlamento-and.es
}

Recibido: 20 de marzo de 2013 Aceptado: 24 de mayo de 2013

\begin{abstract}
Resumen
La financiación de los Grupos parlamentarios de la Cámara andaluza con cargo a su presupuesto desempeña un doble rol. De una parte, es fundamental para el sostenimiento de los Grupos parlamentarios al objeto de establecer y mantener una infraestructura mínima para el desarrollo de las actividades que les son propias y de asistencia a los diputados y diputadas. Por otro lado, contribuye a la financiación de los Partidos políticos bajo cuyas siglas se han presentado a las elecciones. En consecuencia, se hace necesario un control de esta financiación. Este estudio se ocupa de este control desde un punto de vista político, mostrando la actividad desarrollada por la Cámara desde la I Legislatura hasta la actualidad, y que se materializa en los debates habidos, iniciativas parlamentarias presentadas, creación de Comisiones aprobadas, sesiones celebradas, incluso la aprobación de un nuevo Reglamento de la Cámara, todo lo cual, dará una visión real acerca de la preocupación parlamentaria en esta materia.
\end{abstract}

Palabras clave Grupos parlamentarios, financiación partidos políticos, control parlamentario, Parlamento de Andalucía.

\section{The Andalusian Parliament's political control of the parliamentary groups accounting and the funding of the political parties}

\begin{abstract}
The funding of the parliamentary groups in the Andalusian Parliament through their budgets plays a dual role. On one hand, it is essential for the maintenance of the parliamentary groups in order to establish and support an infrastructure for the development of their activities and the assistance to the Members. On the other hand, it contributes to the funding of the political parties under whose initials they have been submitted to the regional elections. Consequently, it is necessary to control this funding. This study addresses this control from a political perspective, showing the activity of the Parliament from the first to the present parliamentary term, activity that is embodied in the debates, parliamentary initiatives submitted, creation of committees, meetings held, including the adoption of a new Regulation of the Parliament, all of which give us a true picture about the parliamentary concern in this matter.
\end{abstract}

Key words

Parliamentary groups; political parties funding; parliamentary control; Parliament of Andalusia. 


\section{INTRODUCCIÓN}

\section{Controversia del asunto}

La financiación de los partidos políticos ha sido desde siempre un tema controvertido y avanza en este sentido, no ya solo en el aspecto doctrinal y legal, sino fundamentalmente en la sociedad y consecuentemente en el foro parlamentario.

Los avances realizados a través de sucesivas reformas en la legislación han ido encaminados a una mayor transparencia en las cuentas de los partidos. Las irregularidades provocadas tanto por los sujetos que realizan las donaciones privadas, como del destino de las mismas, indican la conveniencia de la existencia de un mayor control. Por otro lado, la crisis económica impulsa a introducir mayor austeridad, frente a la necesidad de dinero cada vez mayor para sufragar campañas electorales cada vez más costosas y aparatos políticos cada vez mayores, así como menor dependencia del Estado y de las Comunidades Autónomas.

No obstante lo anterior, a ello se opone una parte interna de los partidos políticos que argumentan la corta duración de las campañas electorales, la financiación escasa de los gastos de funcionamiento ordinario de estos partidos, el importe mínimo de dicha financiación comparada con el montante de los presupuestos del Estado, de la Seguridad Social y de las Comunidades Autónomas, así como el hecho de que es ésta una cuestión general que atañe a todos los países de nuestro entorno.

La financiación pública de los partidos políticos es justificada por sus partidarios diciendo que es la única que garantiza la independencia de los partidos frente a pretensiones externas; la única que garantiza la igualdad mínima de partida para todos y la que hace posible el cumplimiento pleno de su función constitucional.' No obstante, tal como afirma Gaspar Ariño estas tres razones aunque sean ciertamente legítimas, si bien ambivalentes, no son tan claras como a primera vista parece. (Ariño, 2009:21)

\section{Regulación legal}

La Ley Orgánica 8/2007, de 4 de julio, sobre Financiación de los Partidos Políticos, en su artículo 2, bajo la rúbrica "Recursos económicos" establece que los recursos económicos de los partidos políticos estarán constituidos por:

\section{Recursos procedentes de la financiación pública}

a) Las subvenciones públicas para gastos electorales, en los términos previstos en la Ley Orgánica 5/1985, de Régimen Electoral General y en la legisla-

1 Ariño Ortiz, G., “La financiación de los Partidos Políticos” En: Documentos del Foro de la Socie-

236 dad Civil, núm. 1. Madrid: CINCA, 2009, pág.21. 
ción reguladora de los procesos electorales de las Asambleas Legislativas de las Comunidades Autónomas y de las Juntas Generales de los Territorios Históricos vascos.

b) Las subvenciones estatales anuales para gastos de funcionamiento, reguladas en la presente Ley.

c) Las subvenciones anuales que las Comunidades Autónomas establezcan para gastos de funcionamiento en el ámbito autonómico correspondiente, así como las otorgadas por los Territorios Históricos vascos y, en su caso, por las Corporaciones Locales.

d) Las subvenciones extraordinarias para realizar campañas de propaganda que puedan establecerse en la Ley Orgánica reguladora de las distintas modalidades de referéndum.

e) Las aportaciones que en su caso los partidos políticos puedan recibir de los Grupos Parlamentarios de las Cámaras de las Cortes Generales, de las Asambleas Legislativas de las Comunidades Autónomas, de las Juntas Generales de los Territorios Históricos vascos y de los grupos de representantes en los órganos de las Administraciones Locales

\section{Recursos procedentes de la financiación privada}

a) Las cuotas y aportaciones de sus afiliados, adheridos y simpatizantes.

b) Los productos de las actividades propias del partido político y los rendimientos procedentes de la gestión de su propio patrimonio, los beneficios procedentes de sus actividades promocionales, y los que puedan obtenerse de los servicios que puedan prestar en relación con sus fines específicos.

c) Las donaciones en dinero o en especie, que perciban en los términos y condiciones previstos en la presente Ley.

d) Los fondos procedentes de los préstamos o créditos que concierten.

e) Las herencias o legados que reciban.

Se desprende de lo anterior, que un tipo de financiación es la constituida por las aportaciones de las Instituciones representativas. En relación a estas aportaciones, en el caso concreto de la Asamblea Legislativa andaluza, el apartado $3^{\circ}$ del artículo 25 del vigente Reglamento del Parlamento de Andalucía dispone que, los Grupos parlamentarios estarán obligados a llevar una contabilidad específica de las subvenciones parlamentarias que reciban, que pondrán a disposición de la Mesa 
del Parlamento a requerimiento de ésta y, en todo caso, anualmente, antes del 1 de agosto del año siguiente al que la declaración se refiera. La Mesa, con el detalle de presentación que se decida, ordenará su publicación en el Boletín Oficial del Parlamento de Andalucía.

\section{EL CONTROL Y TRANSPARENCIA DE LA FINANCIACIÓN DE LOS PARTIDOS POLÍTICOS EN LA ACTIVIDAD DEL PARLAMENTO DE ANDALUCÍA}

\section{Introducción}

La propia Exposición de Motivos de la Ley Orgánica 8/2007, de 4 de julio, sobre Financiación de los Partidos Políticos explicitó que la Ley Orgánica 3/1987 constituyó la primera regulación de la fuente de ingresos de una de las instituciones básicas sobre la que se sustenta nuestro sistema democrático. Se estima que la regulación que ha de hacer la ley en esta materia, constituye un aspecto nuclear como es la financiación de los partidos políticos, al objeto de garantizar la estabilidad del sistema democrático, así como la transparencia de su actividad económica.

Refleja la sociedad y la ciudadanía un clamor de mayor transparencia y control. Transparencia y control que asciende desde los partidos políticos hasta sus instituciones representativas como son las Cortes Generales y Asambleas parlamentarias de las Comunidades Autónomas.

\section{La financiación de los partidos políticos en los debates de investidura}

Como línea directriz de los que van a ser los temas fundamentales de los que se van a ocupar los Presidentes del Ejecutivo, constituyen los debates de investidura, el instrumento vehicular que sirve de apoyo para ello, de hecho, así lo establece el apartado $3^{\circ}$ del artículo 138 del Reglamento de la Cámara. ${ }^{2}$

A pesar de lo expuesto anteriormente, de los cuatro Presidentes que la Comunidad Autónoma de Andalucía ha tenido al frente de su Ejecutivo, Rafael Rodríguez Escuredo (I Legislatura), José Rodríguez de la Borbolla y Camoyán (I y II Legislaturas), Manuel Chaves González (III a VIII Legislaturas) y José Antonio Griñán Martínez (VIII y IX Legislaturas), únicamente el presidente Manuel Chaves en la IV Legislatura expuso su preocupación por el tema y línea a seguir. ${ }^{3}$ Los restantes, incluido el propio Chaves en el resto de las legislaturas en las que fue investido presidente, obviaron este importante tema. La única cuestión que abordaron relativa a la financiación, fue la autonómica, que es el tema "estrella" desarrollado en todas las sesiones de inves-

2 Artículo 138.3: “A continuación, el candidato o candidata propuesto expondrá, sin limitación de tiempo, el programa político del Gobierno que pretende formar y solicitará la confianza de la Cámara”.

3 DSPA núm. 2, de 19 de julio de 1994, pág. 15 
tidura, acompañado, alguna vez que otra, por financiación de las pymes, de la economía, comunicaciones, empresas, investigación y sanitaria fundamentalmente. ${ }^{4}$

De este modo, el presidente en funciones de la Junta de Andalucía (III Legislatura). y que sería investido para la IV Legislatura (“Legislatura de la pinza”), exponía en su discurso de investidura, celebrado el día 19 de julio de 1994", su intención de alcanzar un "... compromiso institucional basado en un impulso democrático de las instituciones y, al mismo tiempo, en el desarrollo estatutario. Es decir, un compromiso que se plantee a fondo la transparencia de la vida pública y la mayor eficacia de las instituciones, la consagración del Parlamento como centro del debate político, la profundización de la autonomía andaluza y su conversión -la conversión de esta autonomía- en un referente esencial del Estado autonómico español, y plantearnos seriamente la influencia creciente de Andalucía en el proceso de construcción de la Unión Europea."

Para ello, establecía, como premisa básica, para conseguir ese impulso democrático, una total transparencia de la vida pública, de las instituciones y de los partidos políticos. "Los casos de corrupción, la confusión que se ha dado entre intereses públicos y privados o la propia opacidad en la financiación de los partidos políticos han generado un ambiente social de desconfianza. Y ante esta situación sólo sirve un compromiso radical con la democracia, porque es la única fórmula política que nos permite atajar tales conductas."

Abogaba en dicho discurso por una separación entre lo público y lo privado, por unos mecanismos de control y por una transparencia en el funcionamiento de los partidos políticos.

Consecuencia de todo lo anterior, era necesario abordar la financiación de los partidos políticos y su adecuación a las normas de contabilidad general, así como su fiscalización por la Cámara de Cuentas que permitiera un control riguroso de todos sus ingresos y gastos. $Y$ en este sentido, proponía la aprobación de:

a) La creación de una comisión parlamentaria encargada de concretar las normas o compromisos políticos que aseguraran la máxima transparencia en esta financiación.

b) La reforma del Reglamento de la Cámara que debe precisar, debe regular, los mecanismos de control, de agilidad y de transparencia.

4 Concretamente, los temas en materia de financiación fueron autonómica (I, II, IV a IX Legislaturas), de la economía (I y IX Legislaturas), sanitaria (IV y IX Legislatura), de los partidos políticos (IV Legislatura), de las empresas (V Legislatura), de investigación (VI Legislatura), de estructuras de comunicaciones (VII Legislatura), pymes (IX Legislatura). Véase los DSPA núm. 3, de 14 de julio de 1982 (I Legislatura), núm. 45, de 7 de marzo de 1984 (I Legislatura), núm. 2, de 24 de julio de 1986 (II Legislatura), núm. 2, de 23 de julio de 1994 (III Legislatura), núm. 2, de 19 de julio de 1994 (IV Legislatura), núm. 2, de 10 de abril de 1996 (V Legislatura), núm. 2, de 24 de abril de 2000 (VI Legislatura), núm. 2, de 20 de abril de 2004 (VII Legislatura), núm. 2, de 16 de abril de 2008 (VIII Legislatura), núm. 43, de 21 de abril de 2009 (VIII Legislatura) y núm. 2, de 2 de mayo de 2012 (IX Legislatura).

5 DSPA núm. 2, de 19 de julio de 1994 
Respecto a la creación de una comisión parlamentaria encargada de realizar un seguimiento y control de la financiación de los partidos políticos no era un tema nuevo. De hecho, tiene su causa en la III Legislatura, tal como se verá posteriormente. Otra cuestión es, la reforma del Reglamento de la Cámara, por lo que el mismo conllevará una serie de obligaciones a los Grupos parlamentarios de la Cámara en orden a la realización de determinadas contabilidades específicas con las que éstos deben cumplir; tema éste que igualmente abordaré en el último epígrafe. ${ }^{6}$

\section{La Comisión de Seguimiento y Control de la Financiación de los Partidos Políticos}

\section{A. Antecedentes}

Las Comisiones juegan un papel importantísimo en la Cámara legislativa, no solo como órgano preparatorio de los debates y/o leyes que ulteriormente serán debatidos y aprobadas, en su caso, en las sesiones plenarias del Parlamento, sino como entidad propia, como órganos de estudio, investigación, seguimiento y control. Respecto a la financiación de los partidos políticos en el Parlamento de Andalucía, desde la I Legislatura hasta la actualidad (IX Legislatura), han sido ocho las solicitudes de creación de Comisiones que han tenido como objeto la financiación de los partidos políticos; todas ellas, de investigación. De ellas, cinco fueron presentadas en la III Legislatura, una en la V Legislatura y dos en la VIII Legislatura. Es obvio, prima facie, a tenor de estos datos, que fue la III Legislatura la que tuvo más actividad parlamentaria en esta materia; conclusión, que será confirmada si tenemos en cuenta, como veremos posteriormente, que es esta Legislatura el germen de la actual Comisión competente en esta materia y causa de la reforma del Reglamento de la Cámara.

La vida parlamentaria de estas solicitudes de creación de comisiones en materia de financiación de partidos políticos no fue homogénea, sino que tuvieron caminos diferentes que se pueden sintetizar en los siguientes:

— Desfavorables y no admitidas a trámite: 2 (III y VIII Legislatura).

— Retiradas después de calificadas por la Mesa de la Cámara: 2 (III Legislatura).

- Decaídas: 2 (V y VIII Legislatura).

6 Tiene su causa la reforma del Reglamento del Parlamento de Andalucía, entre otros preceptos, en la adición de un nuevo artículo 25.3, que supone la contabilización específica de las subvenciones parlamentarias que reciban, al constituir las mismas una forma de financiación de los partidos políticos, de conformidad con lo prevenido en el artículo 2 de la Ley Orgánica 8/2007, de 4 de julio, sobre Financiación de los Partidos Políticos, que establece en su apartado 1, bajo la rúbrica "Recursos procedentes de la financiación pública”, lo siguiente: “e) Las aportaciones que en su caso los partidos políticos puedan recibir de los Grupos Parlamentarios de las Cámaras de las Cortes Generales, de las Asambleas Legislativas de las Comunida$240 \begin{aligned} & \text { des Autónomas, de las Juntas Generales de los Territorios Históricos vascos y de los grupos de representantes } \\ & \text { en los órganos de las Administraciones Locales" }\end{aligned}$ 
— Rechazadas por el Pleno de la Cámara: 1 (III Legislatura).

- Aprobadas: 1 (III Legislatura).

A mayor abundamiento de lo anterior, de todas las solicitudes de creación de comisiones presentadas en el Registro de la Cámara, únicamente dos de ellas tenían como propósito el estudio, de forma global, de la financiación de los Partidos Políticos y Coaliciones con representación en el Parlamento de Andalucía (sus proponentes eran diversos diputados de los Grupos parlamentarios Socialista e Izquierda Unida Los Verdes-Convocatoria por Andalucía) ${ }^{7}$, mientras que las seis restantes tenían como objeto la investigación y/o esclarecimiento de determinados asuntos concretos relativos a la financiación, especialmente los relativos al Partido Socialista Obrero Español, al ser éste, "el partido mayoritario que apoya al Gobierno andaluz". ${ }^{8}$

El origen de la actual Comisión de Seguimiento y Control de la Financiación de los Partidos Políticos se encuentra precisamente en la solicitud de creación de comisión que fue aprobada, ${ }^{9}$ cuyas conclusiones, de conformidad con lo dispuesto en el apartado $4^{\circ}$ del artículo 52 del Reglamento del Parlamento de Andalucía, se plasmaron en un dictamen que fue discutido en el Pleno de la Cámara junto con los votos particulares presentados. ${ }^{10}$

Esta Comisión, como se verá en el apartado siguiente, extenderá sus trabajos a largo de toda la III Legislatura. En la IV Legislatura, el Pleno del Parlamento de Andalucía, en sesión celebrada los días 18, 19 y 20 de abril de 1995, aprobó la reforma de su Reglamento"1, incorporando como una comisión permanente no legislativa la Comisión de Seguimiento y Control de la Financiación de los Partidos Políticos, y he aquí donde supuestamente hay que encontrar la explicación de la retirada, después de la calificación por la Mesa de la Cámara, de la solicitud de creación de Comisión de Inves-

7 Son las señaladas por los Servicios de la Cámara con los números de expedientes 3-92/CC0006300 (aprobada por el Pleno del Parlamento de Andalucía) y la 4-94/CC-0005288 (retirada después de la calificación y admisión a trámite por la Mesa de la Cámara.

8 Véase la redacción literal de la iniciativa parlamentaria presentada por el Grupo parlamentario Andalucista (Núm. de expediente 3-91/CC-0002206) presentada en la III Legislatura, con fecha 27 de mayo de 1991.

9 Suscrita por diversos diputados del Grupo parlamentario Socialista, presentada en el Registro de la Cámara el día 9 de septiembre de 1992 y aprobada su creación en sesión plenaria del Parlamento de Andalucía el día 15 del citado mes.

10 El texto aprobado por el Pleno del Parlamento de Andalucía, en sesión celebrada los días 30 y 31 de marzo de 1993, en relación con el dictamen de la Comisión de investigación sobre financiación de los partidos políticos y coaliciones con representación en el Parlamento de Andalucía, fue publicado en el BOPA núm. 263, de 20 de abril de 1993, págs. 11288 a 11305, junto con los votos particulares que se mantienen presentados por los Grupos parlamentarios Popular de Andalucía, Izquierda Unida Los Verdes-Convocatoria por Andalucía y Andalucista.

11 El Pleno del Parlamento de Andalucía, en sesión celebrada los días 18, 19 y 20 de abril de 1995, aprobó la reforma de su Reglamento, por el procedimiento de lectura única y quedando incorporada la “Comisión de Seguimiento y Control de la Financiación de los Partidos Políticos con representación en el Parlamento de Andalucía” en el artículo 47.2.7ª y publicada en el BOPA núm 76, de 5 de mayo de 1995, págs. 4329 a 4358 
tigación que proceda al estudio de la financiación de los partidos políticos y coaliciones con representación en el Parlamento de Andalucía, presentada por varios diputados del Grupo parlamentario Izquierda Unida Los Verdes-Convocatoria por Andalucía, explicitada anteriormente, al carecer de sentido con la reforma reglamentaria.

\section{B. La duración de la Comisión}

Concretamente en el punto primero de las conclusiones de la Comisión de Seguimiento y Control de la Financiación de los Partidos Políticos, ésta acordó que seguiría funcionando hasta la terminación de la III legislatura. Es conveniente, respecto a la duración de la Comisión en el ejercicio de las tareas que tenía encomendadas, por acuerdo del Pleno de la Cámara, hacer las siguientes precisiones:

1. Puede suponer un incumplimiento del acuerdo adoptado por el Pleno del Parlamento de Andalucía, en sesión celebrada el día 15 de septiembre de 1992, pues si bien, el mismo especifica que, "Dada su naturaleza, esta Comisión no puede tener una duración determinada..." el mismo establece que "una vez adoptadas conclusiones en alguno de los temas investigados deberán remitirse al Presidente del Parlamento para que con los votos particulares si los hubiere, se proceda a su debate en sesión pública." Por lo que una vez aprobado el susodicho dictamen por el Pleno del Parlamento, la Comisión debería haber finalizado. Recordemos que las comisiones de investigación son aquellas que se crean para un fin concreto y que se extinguen a la finalización del trabajo encomendado; es por tanto una comisión no permanente ${ }^{12}$ a diferencia de las permanentes, las cuales, tendrán dicho carácter durante la legislatura en que el acuerdo se adopte.

2. A mayor abundamiento de lo anterior, es el propio Reglamento del Parlamento de Andalucía el que dispone en el apartado $3^{\circ}$ del artículo 44 que las comisiones deberán concluir la tramitación de cualquier asunto en un plazo máximo de dos meses, excepto en aquellos casos en que el propio Reglamento imponga un plazo distinto o la Mesa de la Cámara, atendidas las circunstancias excepcionales que puedan concurrir, acuerde ampliarlo o reducirlo.

Al haber superado el plazo de dos meses establecidos reglamentariamente, la Mesa de la Cámara debería haber acordado la prórroga de los trabajos de la Comisión, como de hecho, ha sucedido en múltiples ocasiones en casos similares, especialmente con el plazo otorgado a los Grupos de Trabajo o Ponencias de Estudio creados en el seno de alguna Comisión parlamentaria.

3. No obstante lo anterior, la superación del citado límite podría entenderse válido en concordancia con el apartado $2^{\circ}$ del artículo 53 de dicho Reglamento pues

12 Reguladas en la sección tercera del capítulo tercero del título tercero del Reglamento del Parlamento de Andalucía, artículos 52 y ss. 
es la propia Comisión de Investigación la que debe elaborar un plan de trabajo, si bien, es la propia Comisión la que establece una duración limitada, no en función del tiempo transcurrido, sino de las conclusiones adoptadas y elevadas al Pleno, tal como establece el propio dictamen de la Comisión.

4. Como conclusión, hay que entender la omisión del acuerdo por la Mesa de la Cámara únicamente como un aspecto formal ya que es la propia Cámara, reunida en sesión plenaria, la que aprueba la continuación de los trabajos de la Comisión de Investigación. Si bien, “chirría” que sea la propia Comisión la que acordara en su seno, y por tanto, antes del acuerdo del Pleno del Parlamento, su propia continuación, y no realizara una propuesta al mismo, dado que es la Cámara, reunida en sesión plenaria, la competente para adoptar este tipo de acuerdo.

\section{Obligación de los Grupos parlamentarios de llevar una contabilidad}

En el segundo punto de las conclusiones aprobadas por el Pleno del Parlamento, se constató por todos los Grupos parlamentarios la necesidad de llevar unas contabilidades homogéneas y del mismo nivel de concreción que hiciera más racional el estudio comparativo de las mismas. Ese fue un problema con el que la Comisión se encontró en sus trabajos. Para ello, el Pleno del Parlamento de Andalucía acordó solicitar a la Cámara de Cuentas de Andalucía un Plan de Cuentas que, manteniendo una concordancia con los criterios generales del artículo 9 de la Ley Orgánica 3/1987, de 2 de julio, sobre Financiación de los Partidos Políticos y con la normativa del Tribunal de Cuentas del Reino, fuera aplicable con idénticos criterios por todos los Grupos Políticos con representación parlamentaria en la Cámara, en base al artículo $4^{\circ} \mathrm{C}$ de la Ley 1/1988, de 17 de marzo, de la Cámara de Cuentas de Andalucía.

La aprobación de esta conclusión conllevó la reforma del Reglamento del Parlamento de Andalucía en el apartado $3^{\circ}$ del artículo 25; cuestión que es objeto de estudio en el último apartado de este epígrafe.

\section{Obligación de los Grupos parlamentarios de comunicación de abonos}

La cuarta de las conclusiones también supuso otra reforma del Reglamento del Parlamento de Andalucía; en esta ocasión, la adición de un apartado $4^{\circ}$ del artículo 25. Disponía el acuerdo que los Grupos Parlamentarios deberían comunicar anualmente a la Mesa del Parlamento, para su publicación en el BOPA, las cantidades que dichos Grupos abonaran a cada parlamentario en todos sus conceptos, retribuciones, dietas, gastos de representación etc. Los Partidos Políticos debían estar sujetos a un nivel similar de publicidad al de las Administraciones Públicas, rindiendo cuentas a la respectiva Administración que las financiara y, obviamente, remitiendo a la Inspección Tributaría todos los aspectos fiscales por los que han tributado a la Hacienda Pública anualmente. 


\section{E. Reforma del Reglamento del Parlamento de Andalucía}

Concordante con lo expuesto en el último inciso de la letra $A$, y letras $C$ y $D$ de este epígrafe, el Pleno del Parlamento de Andalucía, en sesión celebrada los días 18, 19 y 20 de abril de 1995, aprobó la reforma de su Reglamento, por el procedimiento de lectura única, consecuencia del dictamen de la Comisión de investigación sobre financiación de los partidos políticos y coaliciones con representación en el Parlamento de Andalucía ${ }^{13}$ en el que fueron modificados diversos preceptos. Respecto al tema que se aborda en este estudio, se transcribe a continuación el artículo 25 del Reglamento de la Cámara, una vez modificado su apartado $3^{\circ}$ e introducido el apartado $4^{\circ}$ novo: "1. El Parlamento pondrá a disposición de los Grupos parlamentarios locales y medios materiales suficientes y les asignará, con cargo a su presupuesto, las subvenciones necesarias para cubrir sus gastos de funcionamiento. Las cuantías se fijarán por la Comisión de Gobierno Interior y Derechos Humanos, a propuesta de la Mesa de la Cámara y oídos los portavoces de los Grupos parlamentarios, dentro de los límites de la correspondiente consignación presupuestaria. 2. No obstante lo dispuesto en el punto anterior, el importe de la subvención del Grupo Parlamentario Mixto será establecido atendiendo al número de miembros que compongan dicho Grupo. 3. Los Grupos parlamentarios estarán obligados a llevar una contabilidad específica de las subvenciones parlamentarias que reciban, que pondrán a disposición de la Mesa del Parlamento a requerimiento de ésta, $y$, en todo caso, anualmente, antes del 1 de agosto del año siguiente al que la declaración se refiera. La Mesa, con el detalle de presentación que se decida, ordenará su publicación en el Boletín Oficial del Parlamento de Andalucía. 4. En su caso, los Grupos parlamentarios deberán también comunicar anualmente a la Mesa de la Cámara, antes del 1 de febrero del año siguiente al que la declaración se refiera, las cantidades que abonen a cada parlamentario, cualquiera que fuese su concepto, que serán objeto de publicación en el Boletín Oficial del Parlamento de Andalucía."

De un examen comparativo de este texto con el extinto artículo 25, se pueden realizar las siguientes observaciones:

a) En el anterior artículo $25 \cdot 3,{ }^{14}$ se establecía la obligación por los Grupos parlamentarios de llevar una contabilidad específica de las subvenciones. Con la reforma reglamentaria, el nuevo apartado 3, establece literalmente que deben de llevar " una contabilidad específica de las subvenciones parlamentarias que reciban”. ¿Podría pensarse, por tanto, que deja de existir la obligación para los Grupos parlamentarios de llevar la contabilidad específica de cualquier subvención? Parece que el nuevo reglamento sólo obliga a contabilizar específicamente las provenientes de las asignaciones parlamentarias. Si esto es así, ¿podrían llevar una contabilidad separada del resto de las subvenciones y de los gastos a cargo de aquella? Es decir, podríamos pensar

13 BOPA núm. 76, de 5 de mayo de 1995, págs. 4329 a 4358. DSPA núm. 35, de 18 de abril de 1995, págs. 2036 a 2047.

14 BOPA núm. 60, de 30 de abril de 1991, pág. 2476. 
que únicamente están obligados a contabilizar de forma específica "las subvenciones parlamentarias que reciban”, obviando las demás, si éstas existieran. Asimismo, tendrían únicamente la obligación de aportar los gastos realizados con cargo a las mismas y no todo tipo de gastos efectuados por dichos Grupos parlamentarios.

b) Al amparo del antiguo precepto reglamentario, los Grupos parlamentarios "pondrán a disposición de la Mesa del Parlamento a requerimiento de ésta" la contabilidad específica de las subvenciones. Con el nuevo artículo, se mantiene dicha obligación, pero, además, impone una segunda, y es que "en todo caso, anualmente, antes del 1 de agosto del año siguiente al que la declaración se refiera" [deberán ponerla a disposición de la Mesa]. Con ello, los Grupos parlamentarios deben poner a disposición del citado órgano rector dichas contabilidades antes de la fecha indicada, pero además, también están obligados a entregarlas en cualquier momento, a petición de la Mesa; petición que se entiende que deberá ser expresa.

c) Un último inciso novo de este apartado $3^{\circ}$ es " La Mesa, con el detalle de presentación que se decida, ordenará su publicación en el Boletín Oficial del Parlamento de Andalucía." Cuestión que, como se verá más adelante, es importante, a la vista de la presentación de las contabilidades específicas por los Grupos parlamentarios y que comportará determinadas apreciaciones de forma y de fondo en relación a las mismas, así como la obligación de la Mesa de la Cámara de ordenar su publicación en el BOPA, dado que la publicación es obligatoria al constituir dicho precepto un derecho imperativo y no dispositivo. La única "discrecionalidad" que tiene dicho órgano rector, en este aspecto, es la decisión sobre el detalle de la presentación de la contabilidad, no respecto a su publicación, la cual es preceptiva por la Mesa del Parlamento de Andalucía.

\section{Sesiones celebradas y temas tratados por la Comisión de Seguimiento y Control de la Financiación de los Partidos Políticos con representación en el Parlamento de Andalucía}

\section{A. Sesiones celebradas}

A pesar de la importancia que el asunto tiene, de haber creado una Comisión de Investigación que alargó sus trabajos hasta la finalización de la legislatura en la que fue creada (III Legislatura), de haber aprobado en sesión plenaria de la Cámara las conclusiones del dictamen de la citada Comisión, de haber modificado el Reglamento de la Cámara para introducir una Comisión Permanente cuyo único objeto sea el seguimiento y control de la financiación de los partidos políticos con representación en la Cámara, el número de sesiones celebradas por dicha Comisión a lo largo de todas las legislaturas desde su creación ha sido únicamente 9. Estas sesiones se distribuyen 
en las diferentes legislaturas del siguiente modo: I a III Legislatura: $0^{15}$, IV y V Legislatura: $0^{16}$, VI Legislatura: 1, VII Legislatura: 4, VIII Legislatura: 2, IX Legislatura: 2.

A mayor abundamiento de lo anterior, consultada la base de datos de la Cámara andaluza, y de acuerdo con la misma, se extrae que de las 9 sesiones celebradas por la Comisión de Seguimiento y Control de la Financiación de los Partidos Políticos con representación en el Parlamento de Andalucía, 4 de ellas tuvieron como único objeto la constitución de la Comisión, en cumplimiento de lo previsto en el apartado tercero del artículo 46 del Reglamento de la Cámara. El resto de sesiones, 5, tuvieron como único objeto la elección de algún cargo en la Mesa de la propia Comisión. Se concluye por tanto, que no estudiaron tema alguno de los relativos a su cometido durante todas estas legislaturas. ${ }^{17}$

\section{B. Temas tratados en la Comisión}

En concordancia con lo anterior, las iniciativas parlamentarias, asignadas a la Comisión referida en este epígrafe y objeto de estudio, fueron igualmente escasas, concretamente su número es 12, las cuales se distribuyeron de la siguiente forma: I a IV Legislatura: 0, V Legislatura: 1, VI Legislatura: 1, VII Legislatura: 6, VIII Legislatura: 2, IX Legislatura: 2.

De las 12 iniciativas, 11 fueron por precepto reglamentario, al constituir 7 de ellas elección de los miembros de la Mesa de la Comisión y las 4 restantes por constitución

15 La Comisión de Seguimiento y Control de la Financiación de los Partidos Políticos con representación en el Parlamento de Andalucía es una Comisión permanente creada en la IV Legislatura con ocasión de la reforma del Reglamento de la Cámara, como ha quedado de manifiesto a lo largo de este estudio. Consecuentemente, no existen sesiones anteriores de la misma antes de la legislatura citada. La Comisión referida en la III Legislatura, si bien se denominaba "financiación de los partidos políticos y coaliciones con representación en el Parlamento de Andalucía”, era una Comisión de investigación y no Comisión permanente como la referida en este epígrafe. Por lo que se refiere a las dos primeras legislaturas no existieron comisiones relativas a este asunto.

16 En la IV y V Legislaturas se celebraron sesiones parlamentarias por la Comisión de Seguimiento y Control de la Financiación de los Partidos Políticos con representación en el Parlamento de Andalucía, al menos, por imposición del precepto reglamentario (artículo 46.3 RPA: Las Comisiones Permanentes a que se refieren los apartados anteriores deberán constituirse dentro de los veinte días siguientes a la sesión constitutiva del Parlamento), no obstante, de conformidad con la base de datos del Parlamento de Andalucía, no se refleja que celebraran sesión alguna. Si bien, a pesar de lo anterior, para saber el número exacto de sesiones celebradas podría consultarse las actas de dichas sesiones, aunque las mismas, únicamente estarán a disposición de los Diputados en la Secretaría General de la Cámara, tal como establece el apartado $2^{\circ}$ del artículo 71 del Reglamento de la Cámara.

17 Según consta en la Base de datos del Parlamento de Andalucía, las sesiones celebradas por la Comisión de Seguimiento y Control de la Financiación de los Partidos Políticos con representación en el Parlamento de Andalucía fueron las siguientes: VI Legislatura: 11.05.2000, sesión constitutiva. VII Legislatura: Sesión 19.05.2004, constitutiva. Sesión 18.05.2005, elección de vicepresidente/a y de secretario/a de la Comisión. Sesión 30.11.2005, elección de presidente/a y de secretario/a de la Comisión. Sesión 03.10.2007, elección de secretario/a de la Comisión. VIII Legislatura: Sesión 06.05.2008, constitutiva. Sesión 05.05.2010, elección de vicepresidenta de la Comisión. IX Legislatura: Sesión 28.05.2012, constitutiva. Sesión 26.09.2012,

246 elección de vicepresidente de la Comisión (situación al día 10 de febrero de 2013). 
de la misma, por lo que en puridad, ninguna de ellas constituye, en sí misma, iniciativa parlamentaria a solicitud de ningún grupo parlamentario o diputado. Sólo una iniciativa constituye realmente una actividad parlamentaria en Comisión, es decir, una medida de control parlamentario. Fue en la V Legislatura y tampoco llegó a ser incluida en el orden del día de ninguna sesión de las convocadas correspondientes a la precitada Comisión, al acordar la Mesa de la Cámara su decaimiento por finalización del periodo de sesiones. ${ }^{18}$

Aún más contrasta esta información, si comparamos la única iniciativa parlamentaria presentada ante la Comisión de Seguimiento y Control de la Financiación de los Partidos Políticos con representación en el Parlamento de Andalucía citada en el párrafo anterior, con las 11.086 solicitudes de comparecencias ante comisiones que ha habido en estas legislaturas ${ }^{19}$, lo que hace que represente el 0,0090\% del total de las iniciativas en comisión presentadas. Si las iniciativas parlamentarias suscritas por diputados y grupos parlamentarios representan la inquietud y preocupación por determinados temas y problemas de nuestros representantes en nuestra Comunidad autónoma, podríamos, en consecuencia, ¿deducir la preocupación de la Cámara en materia de financiación de los partidos políticos con representación en el Parlamento de Andalucía?

\section{Otras iniciativas parlamentarias presentadas}

Con independencia de lo anterior, se han presentado en el Parlamento de Andalucía desde su nacimiento, otras iniciativas de diferente tipología como son otros asuntos parlamentarios en Comisión, preguntas orales en pleno, proposiciones no de ley en pleno, solicitudes de información y solicitudes de comparecencia en Comisión diferente a la de Seguimiento y Control de la Financiación de los Partidos Políticos con representación en el Parlamento de Andalucía. Si bien, su número refleja, y a la vez confirma, una apatía política en este tema, que hace ratificar lo expuesto en los párrafos anteriores. Probablemente, si hiciéramos los mismos cálculos estadísticos que los realizados en el epígrafe anterior, el índice referido a las iniciativas relativas a esta materia respecto al total de todas las tipologías aludidas de todas las materias presentadas en la Cámara sería aún inferior.

18 Solicitud de convocatoria 5-96/APC-002402, de la Comisión de Seguimiento y Control de la Financiación de los Partidos Políticos con representación en el Parlamento de Andalucía, para que de manera urgente y a la vista de los hechos denunciados por la Fiscalía Anticorrupción sobre financiación específica en la que aparecen personas vinculadas al PSOE, inicie sus trabajos de investigación estableciendo un calendario de trabajo y de comparecencias, a petición de las Ilmas. Sras. Doña María Amalia Santías Pérez y Doña María Dolores Núñez García del G.p. Popular de Andalucía. La Mesa de la Cámara, en sesión celebrada el día 17 de enero de 2000, acordó el decaimiento después de Mesa por finalización de la $\vee$ Legislatura, de conformidad con lo dispuesto en el artículo 192 del Reglamento del Parlamento, por lo que al final de cada legislatura caducan todos los trámites parlamentarios pendientes de examen y resolución por el Parlamento, excepto aquellos que corresponda conocer a la Diputación Permanente o deban prorrogarse por disposición legal.

19 Consulta realizada el día 13 de febrero de 2013 en base de datos de la Cámara andaluza. (www. parlamentodeandalucia.es) 
Profundizando en ellas, de las 10 presentadas, es la $V$ Legislatura la más prolífica, si se puede decir de aquella que ha tenido el $70 \%$ de las mismas, ya que únicamente se han presentado 1 iniciativa en cada Legislatura III, VI y VIII. Por tipología, destaca la actividad de control realizada en Comisiones como son las comparecencias, seguida de la actividad desarrollada por el Pleno de la Cámara con 3 iniciativas, 2 de las cuales son, a su vez, de control, como son las preguntas orales en pleno, y 1 de impulso político, como son las proposiciones no de ley en pleno. Por último, 2 solicitudes de información dirigidas al Consejo de Gobierno de la Junta de Andalucía. Respecto a los proponentes de la iniciativas, representan el mismo número los pertenecientes a los Grupos parlamentarios Popular de Andalucía e Izquierda Unida Los Verdes-Convocatoria por Andalucía con 4 iniciativas cada uno, 1 a petición del Consejo de Gobierno y por último, 1 a petición de la Presidenta del Tribunal de Cuentas. Para finalizar este epígrafe, diré que un $30 \%$ de las iniciativas presentadas no fueron sustanciadas al ser 2 de ellas decaídas (proposición no de ley en pleno y solicitud de comparecencia en comisión a petición del Grupo parlamentario Popular de Andalucía), es decir, que desde mayo de 1982 (inicio de la I Legislatura) hasta la actualidad, casi 30 años, en la Cámara andaluza únicamente se han sustanciado 7 iniciativas diferentes a la vista en la Comisión referida, de Seguimiento y Control de la Financiación de los Partidos Políticos con representación en el Parlamento de Andalucía, ya relacionadas previamente.

\section{LA FINANCIACIÓN DE LOS GRUPOS PARLAMENTARIOS EN EL PARLAMENTO DE ANDALUCÍA. SUS CONTABILIDADES}

\section{Visión general}

Constituye una vía de financiación de los partidos políticos la formada por las aportaciones que, en su caso, estos partidos puedan recibir de los Grupos Parlamentarios de las Asambleas Legislativas de las Comunidades Autónomas, de conformidad con lo prevenido en el artículo 2 de la Ley Orgánica 8/2007, de 4 de julio, sobre Financiación de los Partidos Políticos, el cual, establece en su apartado 1, bajo la rúbrica "Recursos procedentes de la financiación pública", lo siguiente: "e) Las aportaciones que en su caso los partidos políticos puedan recibir de los Grupos Parlamentarios de las Cámaras de las Cortes Generales, de las Asambleas Legislativas de las Comunidades Autónomas, de las Juntas Generales de los Territorios Históricos vascos y de los grupos de representantes en los órganos de las Administraciones Locales". En el caso del Parlamento de Andalucía, el artículo 25 del Reglamento de la Cámara establece, en su apartado $3^{\circ}$, que los Grupos parlamentarios estarán obligados a llevar una contabilidad específica de las subvenciones parlamentarias que reciban, las cuales serán objeto de publicación en el Boletín Oficial del Parlamento de Andalucía.

En 1994 comienza a publicarse las citadas contabilidades, si bien, éstas no se publican en virtud de dicho artículo 25.3 del Reglamento del Parlamento, sino como recoge la resolución de la Presidencia de la Cámara, contenida en la orden de publicación, es

248 "en cumplimiento de los Acuerdos adoptados en Pleno, en sesión celebrada el zo de marzo 
de 1993, con ocasión de la aprobación de las conclusiones de la Comisión de Investigación sobre la Financiación de los Partidos Políticos y Coaliciones con representación en esta Cámara" así como por Resolución de 29 de junio de 1993 por el que se dispone la publicidad de la contabilidad de las subvenciones recibidas de la Cámara andaluza por sus Grupos parlamentarios. A pesar de la prontitud con que es cumplida la voluntad del Parlamento de Andalucía, reflejada en dichos acuerdos, también de forma vertiginosa, dicha voluntad será convertida en un acto de trámite, visto el escaso interés real en homogeneizar las contabilidades para hacer "más racional el estudio comparativo de las mismas"ro. Reflejo de ello, es el incumplimiento del precepto reglamentario referido en tanto que, las contabilidades de los Grupos parlamentarios correspondientes a los ejercicios 2002 y 2004 no fueron publicadas. Pero más allá de este incumplimiento, cuyas causas se desconocen, es habitual en la publicación de estas contabilidades la falta de homogeneidad ya referida, que se refleja, a efectos ilustrativos de lo dicho, en la presentación, por algunos Grupos parlamentarios (véase, por ejemplo, el Grupo parlamentario Izquierda Unida Los Verdes-Convocatoria por Andalucía), del balance de situación unas veces sí y otras veces no. En el mismo sentido, las cuentas de resultados son presentadas unas veces con bastante detalle, otras son reflejadas de forma muy sucinta, hasta incluso se llega el extremo de que existen codificaciones que no aparecen.

En términos parecidos, se puede predicar del Grupo parlamentario Popular de Andalucía, que en ocasiones desciende a detalles y otras se limita a unos epígrafes globales. Respecto al Grupo parlamentario Socialista, es más homogéneo en cuánto a la unicidad de criterio en la presentación de sus contabilidades a lo largo de los años; no obstante, es necesario destacar lo parco en los detalles reflejados en su contabilidad, pues es habitual que únicamente exponga un resumen de cada codificación, sin descender nunca a mayor explicitación: hechos que son constatables a partir de la contabilidad del ejercicio correspondiente a 2010.

Consecuente con todo lo anterior, se puede afirmar la carencia de exigencia de unicidad de criterio por parte de la Mesa de la Cámara, respecto a la publicación que ordena, pues cada año suele publicar las contabilidades con niveles de concreción diversos, lo que evidencia que no exige ningún nivel de concreción determinado, limitándose a la mera publicación de lo aportado por los Grupos parlamentarios. ${ }^{21}$ Sería aconsejable, por tanto, un acuerdo que exigiera un nivel de detalles y codificación que fuera común en la presentación de las contabilidades de los Grupos parlamentarios. Avala esta tesis, la orden de publicación, realizada por la Mesa de la Cámara, en el año

20 Tal como recoge literalmente la conclusión segunda del texto aprobado por el Pleno del Parlamento de Andalucía en sesión celebrada el 30 de marzo de 1993, con ocasión de la aprobación de las conclusiones de la Comisión de Investigación sobre la Financiación de los Partidos Políticos y Coaliciones con representación en esta Cámara. BOPA núm. 263, de 20 de abril de 1993, pág. 11304

21 Ejemplo de lo expuesto, es la contabilidad correspondiente al ejercicio de 1999, por ser bastante sucinta y escueta de forma global por todos los Grupos parlamentarios (aparecen entre 4 y 10 líneas en la cuenta de pérdidas y ganancias y otras tantas en el balance de situación, dependiendo del Grupo parlamentario autor). Sin embargo, y precisamente por ello, supuestamente, presentan dichas contabilidades un grado alto de homogeneidad común a todos los Grupos parlamentarios. 
2002, de las contabilidades específicas de las subvenciones parlamentarias recibidas correspondientes al ejercicio de 2001, mezclando las mismas los importes expresados en euros y en pesetas; de este modo, se puede constatar como las contabilidades publicadas correspondientes a los Grupos parlamentarios Mixto e Izquierda Unida Los Verdes-Convocatoria por Andalucía se reflejan en euros, mientras que las presentadas por los Grupos parlamentarios Popular de Andalucía y Socialista se reflejan en pesetas.

Acentuando aún más, si cabe, lo expuesto en los párrafos anteriores, se publica incluso la contabilidad aportada por el Grupo parlamentario Andalucista, tal como es entregada por el mismo, sin solicitar subsanación de errores por la Mesa de la Cámara, dado que, el citado Grupo refleja sus gastos (gastos de personal, financieros, tributos, amortizaciones...) como ingresos y en la columna del debe de la cuenta de resultados, mientras que debería aparecer como gastos en la columna del debe como gastos. Por otro lado, las asignaciones, que les entrega el Parlamento de Andalucía, ni las refleja como ingreso, ni como gasto, sino que las recoge como concepto aparte.

Expongo, a continuación, diversos aspectos de las contabilidades de los Grupos parlamentarios, publicadas en el BOPA, si bien, los cuadros son de elaboración propia, los importes son extraídos de las contabilidades de los Grupos, tal como recogen las publicaciones en el Boletín durante los años 1994 a 2012 (salvo las referidas a los años 2003 y 2005 correspondientes a los ejercicios económicos 2002 y 2004, respectivamente), estando pendiente la publicación correspondiente a la contabilidad del ejercicio de 2012, cuya publicación será a partir del día 1 de agosto de $2013 . .^{22}$ Igualmente, es necesa-

22 BOPA núm. 7, de 2 de agosto de 1994, págs. 81 a 86 (Contabilidad de las subvenciones puestas a disposición de cada Grupo Parlamentario por el Parlamento de Andalucía durante el año 1993); BOPA núm. 112, de 26 de septiembre de 1995, págs. 5778 a 5784 (Contabilidad de las subvenciones puestas a disposición de cada Grupo Parlamentario por el Parlamento de Andalucía durante el año 1994); BOPA núm. 44, de 27 de septiembre de 1996, págs. 4172 a 4177 (Contabilidad de las subvenciones puestas a disposición de cada Grupo Parlamentario por el Parlamento de Andalucía durante el año 1995); BOPA núm. 145, de 19 de septiembre de 1997, págs. 9319 a 9320 (Contabilidad de las subvenciones puestas a disposición de cada Grupo Parlamentario por el Parlamento de Andalucía durante el año 1996); BOPA núm. 244, de 21 de agosto de 1998, págs. 14066 a 14068 (Contabilidad de las subvenciones puestas a disposición de cada Grupo Parlamentario por el Parlamento de Andalucía durante el año 1997); BOPA núm. 356, de 17 de agosto de 1999, págs. 20886 a 20887 (Contabilidad de las subvenciones puestas a disposición de cada Grupo Parlamentario por el Parlamento de Andalucía durante el año 1998); BOPA núm. 47, de 22 de septiembre de 2000, págs. 2279 a 2282 (Contabilidad de las subvenciones puestas a disposición de cada Grupo Parlamentario por el Parlamento de Andalucía durante el año 1999); BOPA núm. 176, de 21 de agosto de 2001, págs. 9387 a 9388 (Contabilidad de las subvenciones puestas a disposición de cada Grupo Parlamentario por el Parlamento de Andalucía durante el año 2000); BOPA núm. 213, de 16 de noviembre de 2001, págs. 11874 a 11875 (Corrección de errores de la publicación anterior); BOPA núm. 380, de 24 de septiembre de 2002, págs. 21393 a 21394 (Contabilidad de las subvenciones puestas a disposición de cada Grupo Parlamentario por el Parlamento de Andalucía durante el año 2001); BOPA núm. 49, de 10 de agosto de 2004, págs. 3351 a 3352 (Contabilidad de las subvenciones puestas a disposición de cada Grupo Parlamentario por el Parlamento de Andalucía durante el año 2003); BOPA núm. 536, de 26 de octubre de 2006, págs. 29371 a 29373 (Contabilidad específica de las subvenciones parlamentarias recibidas por los Grupos parlamentarios durante el año 2005); BOPA 772, de 3 de diciembre de 2007, págs. 41100 a 41102 (Contabilidad específica de las subvenciones parlamentarias recibidas por los Grupos parlamentarios durante el año 2006); BOPA núm. 
rio precisar que los datos reflejados en los cuadros siguientes, referidos a los años 1993 a 2001, son obtenidos una vez convertidos los importes publicados en pesetas a euros.

\section{Las subvenciones parlamentarias recibidas por los Grupos parlamentarios con cargo al presupuesto del Parlamento de Andalucía ${ }^{23}$}

Expongo a continuación el cuadro 1, expresivo de los importes totales recibidos por los Grupos parlamentarios con cargo al presupuesto del Parlamento de Andalucía, haciendo constar que las publicaciones realizadas en el BOPA se realizan anualmente y que, por tanto, la columna "TOTAL" está formada por la suma de las subvenciones recibidas por los Grupos en un ejercicio económico:

\section{CUADRO 1}

\begin{tabular}{|c|c|c|c|c|c|}
\hline & Socialista & Popular & IULV-CA & Andalucista & TOTAL* \\
\hline 1993 & $3.059 .040,62 €$ & $1.538 .963,61 €$ & $803.727,48 €$ & $536.449,98 €$ & $5.938 .181,69 €$ \\
\hline 1994 & $2.739 .421,15 €$ & $1.905 .627,42 €$ & $1.090 .788,67 €$ & $357.573,32 €$ & $6.093 .410,56 €$ \\
\hline 1995 & $2.207 .879,61 €$ & $2.063 .033,89 €$ & $1.155 .947,28 €$ & $364.511,95 €$ & $5.791 .372,73 €$ \\
\hline 1996 & $2.514 .163,33 €$ & $2.075 .093,08 €$ & $951.712,26 €$ & $410.268,50 €$ & $5.951 .237,18 €$ \\
\hline 1997 & $2.788 .056,81 €$ & $2.091 .316,94 €$ & $817.507,12 €$ & $401.182,79 €$ & $6.098 .063,66 €$ \\
\hline 1998 & $2.852 .378,33 €$ & $2.278 .708,29 €$ & $880.349,28 €$ & $420.775,79 €$ & $6.432 .211,68 €$ \\
\hline 1999 & $2.963 .225,22 €$ & $2.305 .017,48 €$ & $884 \cdot 308,10 €$ & $421.885,88 €$ & $6.574 .436,69 €$ \\
\hline 2000 & $2.906 .699,99 €$ & $2.535 .787,39 €$ & $597 \cdot 941,29 €$ & $461.472,14 €$ & $6.501 .900,81 €$ \\
\hline 2001 & $3.003 .663,55 €$ & $2.686 .659,48 €$ & $530.812,78 €$ & $423.047,68 €$ & $6.644 .183,50 €$ \\
\hline 2002 & - & - & - & - & - \\
\hline 2003 & $3.334 .426,92 €$ & $2.980 .469,88 €$ & $578.346,00 €$ & $393.179,16 €$ & $7.286 .421,96 €$ \\
\hline 2004 & - & - & - & - & - \\
\hline 2005 & $4.804 .416,96 €$ & $3.068 .847,88 €$ & $721.836,60 €$ & $699.835,97 €$ & $9.294 .937,41 €$ \\
\hline 2006 & $5.189 .681,74 €$ & $3.313 .859,98 €$ & $802.260,86 €$ & $701.383,72 €$ & $10.007 .186,30 €$ \\
\hline 2007 & $5.618 .821,60 €$ & $3.586 .969,44 €$ & $865.624,48 €$ & $810.900,82 €$ & $10.882 .316,34 €$ \\
\hline 2008 & $5.178 .131,80 €$ & $4.188 .746,74 €$ & $986.418,03 €$ & $139.457,95 €$ & $10.492 .754,52 €$ \\
\hline 2009 & $5.624 .828,54 €$ & $4.762 .337,53 €$ & $1.086 .781,68 €$ & & $11.473 .947,75 €$ \\
\hline 2010 & $5.286 .261,92 €$ & $4.477 .862,00 €$ & $1.049 \cdot 370,64 €$ & & $10.813 .494,56 €$ \\
\hline 2011 & $5.618 .286,02 €$ & $4.755 .795,01 €$ & $1.082 .169,24 €$ & & $11.456 .250,27 €$ \\
\hline
\end{tabular}

* No se computan las asignaciones del G.p. Mixto al carecer de importancia a los efectos de financiación de los partidos políticos. En cualquier caso, solo se han publicado en el Boletín Oficial del Parlamento de Andalucía, relativos a este grupo parlamentario, los pertenecientes a los años 2001, 1993 y 1994.

págs. 10 a 13 (Contabilidad específica de las subvenciones parlamentarias recibidas por los Grupos parlamentarios durante el año 2008); BOPA núm. 517, de 9 de septiembre de 2010 (Contabilidad específica de las subvenciones parlamentarias recibidas por los Grupos parlamentarios durante el año 2009); BOPA núm. 749, de 26 de septiembre de 2011, págs. 10 a 12 (Contabilidad específica de las subvenciones parlamentarias recibidas por los Grupos parlamentarios durante el año 2010); BOPA núm. 72, de 27 de septiembre de 2012, págs. 4 a 7 (Contabilidad específica de las subvenciones parlamentarias recibidas por los Grupos parlamentarios durante el año 2011).

23 Aparecen con el código 74. 
No es mi intención realizar comparaciones interminables y comentarios gratuitos respecto a los datos anteriores, que puede realizar e interpretar el lector, pero sí creo conveniente destacar, únicamente, el incremento de las subvenciones realizadas por el Parlamento de Andalucía a sus Grupos parlamentarios, habiéndose aumentadas las mismas en un 92,92 \% desde el año 1993 a 2011.

\section{Las aportaciones realizadas por los Grupos parlamentarios a sus organizaciones y/o partidos políticos bajo cuyas siglas se presentaron a las elecciones}

Si las subvenciones parlamentarias recibidas por los Grupos parlamentarios con cargo al presupuesto del Parlamento de Andalucía aparecen recogida por los mismos de forma completa, no puede decirse lo mismo si nos referimos a las aportaciones realizadas por estos Grupos a sus organizaciones y/o partidos políticos bajo cuyas siglas se presentaron a las elecciones. Lo habitual, es que la expresión de dichos importes venga reflejada en el código 65 (CUADRO 2), si bien, podrá comprobarse como el Grupo parlamentario Socialista, ${ }^{24}$ no tenía el mismo criterio antes de 2003 , por lo que desde los años comprendidos desde 1993 a 2001, este Grupo parlamentario no refleja en este código las entregas a su organización política. Deduzco, a la vista de los importes consignados, que durante esos intervalos, vienen recogidos bajo el código 62, correspondiente a servicios exteriores y gastos de explotación. De hecho, dichos importes reflejan una línea de regularidad ascendente, compatible con dicho concepto, bajando bruscamente a partir de 2003 , ejercicio en el que incorpora dichas aportaciones al código 65 "Entrega a sus organizaciones, partidos...".

Como consecuencia de lo anterior, es necesario para una comprensión global de las aportaciones realizadas por los Grupos parlamentarios a sus organizaciones y/o partidos políticos bajo cuyas siglas se presentaron a las elecciones, aportar otro cuadro más (CUADRO 3), que comprende los importes bajo el concepto de servicios exteriores y gastos de explotación.

Quiero precisar que en el año 1994, el Grupo parlamentario Izquierda Unida Los Verdes-Convocatoria por Andalucía, recoge en un código denominado 67 "Gastos extraordinarios" el importe de 68.201.612 pesetas (409.899,94 $€$ ); importe que, sin embargo, se recoge en este cuadro, al entender que pudiera ser aportación a su coalición política, pues no recoge en ninguna otra partida tal finalidad y es difícil pensar en gastos extraordinarios de semejante importe. Ello es corroborado con la cantidad reflejada en 1993 por el citado Grupo parlamentario de 83.457 .063 pesetas, que a pesar de indicar también el mismo código 67, expresa, sin embargo, la denominación de "aportación a IU-CA".

24 En el mismo sentido, aunque en menor medida, también el Grupo parlamentario Izquierda Unida Los Verdes-Convocatoria por Andalucía, utiliza criterios diversos respecto a las asignaciones referidas a varios ejercicios económicos. 
CUADRO 2

\begin{tabular}{|c|c|c|c|c|c|}
\hline & Socialista & Popular & IULV-CA & Andalucista & TOTAL \\
\hline 1993 & No aparece & $781.116,37 €$ & $501.587,05 €$ & No aparece & $1.282 .703,42 €$ \\
\hline 1994 & No aparece & $1.205 .478,91 €$ & $409.899,94 €$ & No aparece & $1.615 .378,85 €$ \\
\hline 1995 & No aparece & $1.474 .684,65 €$ & No aparece & $270.496,48 €$ & $1.745 .181,13 €$ \\
\hline 1996 & No aparece & $1.677 .500,61 €$ & No aparece & No aparece & $1.677 .500,61 €$ \\
\hline 1997 & No aparece & No aparece & $194.032,48 €$ & No aparece & $194.032,48 €$ \\
\hline 1998 & No aparece & $1.926 .243,79 €$ & No aparece & No aparece & $1.926 .243,79 €$ \\
\hline 1999 & No aparece & $1.914 .223,55 €$ & $133.584,39 €$ & No aparece & $2.047 .807,94 €$ \\
\hline 2000 & No aparece & $1.995 .360,19 €$ & $63.425,17 €$ & No aparece & $2.058 .785,35 €$ \\
\hline 2001 & No aparece & $1.982 .738,93 €$ & $104.045,84 €$ & No aparece & $2.086 .784,77 €$ \\
\hline 2002 & - & - & - & - & - \\
\hline 2003 & $3.017 .414,09 €$ & $2.250 .000,00 €$ & No aparece & No aparece & $5.267 .414,09 €$ \\
\hline 2004 & - & - & - & & - \\
\hline 2005 & $4.396 .225,46 €$ & $2.160 .500,00 €$ & No aparece & No aparece & $6.556 .725,46 €$ \\
\hline 2006 & $4.735 .416,74 €$ & $2.754 .000,00 €$ & No aparece & No aparece & $7.489 .416,74 €$ \\
\hline 2007 & $5.177 .843,55 €$ & $2.964 .000,00 €$ & No aparece & No aparece & $8.141 .843,55 €$ \\
\hline 2008 & $4.765 .833,63 €$ & $3.526 .000,00 €$ & No aparece & No aparece & $8.291 .833,63 €$ \\
\hline 2009 & $5.182 .196,39 €$ & $4.100 .000,00 €$ & No aparece & & $9.282 .196,39 €$ \\
\hline 2010 & $4.846 .191,72 €$ & $3.792 .000,00 €$ & $1.420,21 €$ & & $8.639 .611,93 €$ \\
\hline 2011 & $5.134 .706,55 €$ & $4.040 .000,00 €$ & $124.116,18 €$ & & $9.298 .822,73 €$ \\
\hline & & & & & \\
\hline 1964 & & & & - \\
\hline
\end{tabular}

Es el Grupo parlamentario Popular de Andalucía el que de forma más regular expresa estas cantidades, salvo en la contabilidad correspondiente al ejercicio económico de 1997, sin que tampoco se vea incrementado el importe de "servicios exteriores".

Es, en cambio, el Grupo Parlamentario Andalucista, el que, salvo el ejercicio de 1995, no refleja jamás las cantidades aportadas a su partido político y/o coalición. No obstante lo anterior, si relacionamos los cuadros 2 y 3, observamos en el cuadro 3 "servicios exteriores" que aparecen siempre asignaciones todos los años, excepto en el año 1995 que es el único, como se ha dicho anteriormente, que si aparece en el código relativo a las "asignaciones a partidos". 25

25 Se publica erróneamente en el BOPA núm. 297, de 15 de septiembre de 2009, pág. 11, al aparecer recogido con la codificación 64, como si se tratase de gastos de personal, en lugar de la codificación 62 que es la adecuada. 
CUADRO 3

\begin{tabular}{|c|c|c|c|c|c|}
\hline & Socialista & Popular & IULV-CA & Andalucista & TOTAL \\
\hline 1993 & $1.320 .811,08 €$ & $155.647,36 €$ & $28.197,73 €$ & $57.555,32 €$ & $1.562 .211,49 €$ \\
\hline 1994 & $1.201 .029,64 €$ & $72.031,66 €$ & $372.720,50 €$ & $29.812,72 €$ & $1.675 .594,52 €$ \\
\hline 1995 & $1.278 .815,64 €$ & $99.129,69 €$ & $255.905,82 €$ & No aparece & $1.633 .851,15 €$ \\
\hline 1996 & $1.648 .260,68 €$ & $104.455,01 €$ & $180.335,12 €$ & $16.934,22 €$ & $1.949 .985,04 €$ \\
\hline 1997 & $1.845 .688,24 €$ & $151.927,93 €$ & $166.975,27 €$ & $37.331,00 €$ & $2.201 .922,44 €$ \\
\hline 1998 & $1.678 .066,69 €$ & $164.280,73 €$ & $190.606,79 €$ & $42.174,90 €$ & $2.075 .129,11 €$ \\
\hline 1999 & $2.769 .683,83 €$ & $213.805,50 €$ & $109.691,10 €$ & $42.123,31 €$ & $3.135 .303,74 €$ \\
\hline 2000 & $2.677 .431,24 €$ & $236.446,91 €$ & $115.249,80 €$ & $2.846,35 €$ & $3.031 .974,31 €$ \\
\hline 2001 & $2.721 .233,25 €$ & $248.664,00 €$ & $100.740,10 €$ & $379,61 €$ & $3.071 .016,96 €$ \\
\hline 2002 & - & - & - & - & - \\
\hline 2003 & $67.390,95 €$ & $182.051,42 €$ & $85.973,35 €$ & No aparece & $335.415,72 €$ \\
\hline 2004 & - & - & - & - & - \\
\hline 2005 & $37.057,12 €$ & $240.978,74 €$ & $91.914,48 €$ & $16.113,64 €$ & $386.063,98 €$ \\
\hline 2006 & $78.028,61 €$ & $249.223,66 €$ & $166.215,32 €$ & $30.674,63 €$ & $524.142,22 €$ \\
\hline 2007 & $39.932,88 €$ & $195.034,54 €$ & $192.482,55 €$ & $40.178,90 €$ & $467.628,87 €$ \\
\hline 2008 & $39.247,22 €$ & $194.645,13 €$ & $210.751,38 € 2$ & $27.232,46 €$ & $471.876,19 €$ \\
\hline 2009 & $46.680,86 €$ & $213.809,54 €$ & $269.094,88 €$ & & $529.585,28 €$ \\
\hline 2010 & $42.606,72 €$ & $211.187,98 €$ & $352.560,44 €$ & & $606.355,14 €$ \\
\hline 2011 & $55.506,32 €$ & $223.051,59 €$ & $372.817,41 €$ & & $651.375,32 €$ \\
\hline
\end{tabular}

Respecto al resto de los importes reflejados en el cuadro 3, no citados anteriormente, podemos sospechar que muchos de ellos son asignaciones a sus partidos y coaliciones con las cuales acudieron a las elecciones al Parlamento de Andalucía, pero de los que no hay constancia de ello según la información de las contabilidades publicadas y puestas a disposición de la Mesa de la Cámara; cuestión diferente es la documentación aportada por los propios Partidos políticos al objeto de su control por el órgano fiscalizador.

\section{Conclusiones}

I. A pesar de lo que pudiera entenderse de la literalidad del artículo 25.3, los Grupos parlamentarios han optado por llevar una contabilidad general de todas las subvenciones recibidas así como de otros ingresos aportados, lo que, a mi juicio, supera lo dispuesto en el precepto citado, ya que este únicamente establece que los mismos deben aportar "una contabilidad específica de las subvenciones parlamentarias que reciban".

II. Con el vigente reglamento de la Cámara, los Grupos parlamentarios deben 254 poner a disposición de la Mesa del Parlamento la contabilidad específica de las sub- 
venciones parlamentarias que reciban, y, en todo caso, anualmente, antes del 1 de agosto del año siguiente al que la declaración se refiera, si bien, también pondrán a disposición de la Mesa dichas contabilidades a requerimiento de ésta. Respecto a este punto, caben realizar dos observaciones:

a. No consta que la Mesa de la Cámara haya ejercido alguna vez la facultad de requerir a los Grupos parlamentarios la contabilidad específica de las subvenciones parlamentarias fuera de los periodos obligatorios a los que están sometidos los Grupos parlamentarios.

b. A pesar de que la reforma del reglamento parlamentario, en lo que afecta al artículo 25.3, se realizó por acuerdo aprobado por el Pleno del Parlamento de Andalucía, en sesión celebrada los días 18, 19 y 20 de abril de 1995, publicada en BOPA núm. 76, de 5 de mayo de 1995 y cuya entrada en vigor fue el mismo día de su publicación en el citado boletín, en virtud de su disposición final, sin embargo, la Mesa no ordenó la publicación de las contabilidades específicas correspondientes a los años 2002 y 2004, pese a estar obligada a ello. No se tiene constancia de si, al menos, conoció las citadas contabilidades, o simplemente no las publicó, o incluso si los Grupos parlamentarios incumplieron con su deber de ponerlas a disposición de la Mesa de la Cámara antes del 1 de agosto de los años siguientes a los que las declaraciones se refieren. En cualquier caso, es manifiesto el incumplimiento reglamentario realizado por la propia Mesa del Parlamento de Andalucía.

III. El último inciso del apartado $3^{\circ}$ del susodicho artículo 45 dispone que " $L a$ Mesa, con el detalle de presentación que se decida, ordenará su publicación en el Boletín Oficial del Parlamento de Andalucía." A tenor de lo dispuesto anteriormente, parece deducible y meridianamente evidente, que la Mesa no ha adoptado acuerdo alguno en relación con el detalle de presentación con que los Grupos parlamentarios deben poner sus contabilidades específicas a disposición de la misma; cuestión que, hubiera sido más que deseable, a la luz de la publicación de dichas contabilidades. Incluso parece que la Mesa se limita a ordenar su publicación sin ni siquiera entrar a conocer su contenido, no ya de fondo, sino al menos, en su forma, homogeneidad en la presentación, así como la profundidad o el carácter somero de dichas contabilidades, ya que las mismas varían considerablemente entre las correspondientes a unos Grupos parlamentarios y otros, así como entre unos años y otros realizadas por un mismo Grupo parlamentario. Entiendo, a la vista del precepto estudiado, así como desde el prisma de una óptima aplicación jurídica, que la Mesa tiene la potestad de decidir el detalle de presentación, pero tiene, a su vez, la obligación de tomar la decisión en cuanto al grado de detalle. Es decir, ha de adoptar, al menos, un acuerdo en relación con la presentación; no obstante, tiene la potestad para decidir sobre el contenido del mismo.

A mayor abundamiento de lo expuesto, si bien es cierto, que no parece que sea función de la Mesa calificar, ni adoptar acuerdo alguno, en base a la admisión a trámi- 
te de este tipo de documento, si le corresponde a dicho órgano rector, a mi juicio, al amparo de lo dispuesto en el artículo $28.1^{\circ}$ del Reglamento de la Cámara, adoptar cuantas decisiones y medidas requieran la organización del trabajo. Y es precisamente, en base a este precepto, y teniendo como finalidad la transparencia, atendiendo a las conclusiones de la Comisión de investigación, cuyo dictamen fue aprobado por la Cámara, en sesión plenaria ${ }^{26}$, y fundamentalmente al espíritu de la misma, y a cuyos efectos se reformó el artículo 25.3 del Reglamento del Parlamento, por lo que hubiera sido deseable que la Mesa del Parlamento de Andalucía hubiera adoptado alguna medida en orden al esclarecimiento y homogeneidad de las citadas contabilidades. De hecho, en la conclusión $2^{\text {a }}$ del citado dictamen ${ }^{27}$ se establecía que se había constatado por todos los Grupos parlamentarios "la necesidad de llevar unas contabilidades homogéneas y del mismo nivel de concreción que hiciera más racional el estudio comparativo de las mismas". Ese fue "un problema con el que la Comisión se encontró en [sus] trabajos". Para ello, el Pleno del Parlamento de Andalucía acordó "solicitar a la Cámara de Cuentas de Andalucía un Plan de Cuentas que, manteniendo una concordancia con los criterios generales del artículo 9 de la Ley Orgánica 3/1987, de 2 de julio, sobre Financiación de los Partidos Políticos y con la normativa del Tribunal de Cuentas del Reino, fuera aplicable con idénticos criterios por todos los Grupos Políticos con representación parlamentaria en [la] Cámara".

IV. Consecuencia de todo lo anterior, es que en los veinte años transcurridos desde entonces, las contabilidades puesta a disposición por los Grupos parlamentarios, y publicadas en el BOPA, parecen que no han variado sustancialmente en cuánto a los problemas que ya adolecían antes de 1993, pues si la Comisión de Investigación, que elaboró dicho dictamen, se encontró con serios problemas en el trabajo que el Pleno de la Cámara le había encargado, en la actualidad, continúan las mismas dificultades para realizar un estudio comparativo de las mismas, al no existir el mismo nivel de concreción, ni ser las contabilidades homogéneas, ni tener los Grupos parlamentarios un concepto inequívoco de los gastos, no ya común a todos ellos, sino ni siquiera en un mismo Grupo a través del trascurso de los años, pues el criterio empleado por un mismo Grupo parlamentario va transformándose con el paso del tiempo. Baste citar, a efectos ilustrativos, como las aportaciones realizadas por algunos Grupos parlamentarios a los Partidos políticos, bajo cuyas siglas acudieron a las elecciones, aparecen reflejadas unas veces como "subvenciones a la organización" y otras como "servicios externos" con codificación diferente.

\section{BIBLIOGRAFÍA}

Ariño Ortiz, G. (2009), “La financiación de los Partidos Políticos” En: Documentos del Foro de la Sociedad Civil, núm. 1. Madrid: CINCA

25626 BOPA núm. 263, de fecha 20 de abril de 1983, págs. 11288 a 11305

27 BOPA núm. 263, ibíd., pág. 11294 
Artés Caselles, J. y García Viñuela, E. (2009), “La economía política de las reformas de la financiación electoral”. En: Revista Española de Ciencia Política, Madrid: Asociación.

Catalunya Sindicatura de Comptes (2010), Acuerdo de transparencia y autolimitación de gastos electorales y de financiación de los partidos políticos. Ejercicios 20032006. Barcelona: La Sindicatura

Mirón Ortega, M.A. (2001), "Subvenciones y contabilidad de los grupos parlamentarios". En: Corts: Anuario de Derecho Parlamentario. Valencia: Publicaciones de las Cortes Valencianas, 10:180-203 